\title{
Les prénoms masculins à Rennes pendant la révolution (1785-1805)
}

Jean-Pierre Lethuillier

\section{(2) OpenEdition \\ 12 Journals}

Édition électronique

URL : https://journals.openedition.org/ahrf/128

DOI : $10.4000 /$ ahrf.128

ISSN : 1952-403X

Éditeur :

Armand Colin, Société des études robespierristes

Édition imprimée

Date de publication : 1 décembre 2000

Pagination : 87-110

ISSN : 0003-4436

Référence électronique

Jean-Pierre Lethuillier, «Les prénoms masculins à Rennes pendant la révolution (1785-1805) »,

Annales historiques de la Révolution française [En ligne], 322 | octobre-décembre 2000, mis en ligne le 21 février 2006, consulté le 22 avril 2022. URL : http://journals.openedition.org/ahrf/128 ; DOI : https:// doi.org/10.4000/ahrf.128

Ce document a été généré automatiquement le 22 avril 2022.

Tous droits réservés 


\title{
Les prénoms masculins à Rennes pendant la révolution (1785-1805)
}

\author{
Jean-Pierre Lethuillier
}

1 Cette enquête ne porte pas sur les seuls "prénoms révolutionnaires" mais sur l'ensemble du corpus des prénoms en usage à Rennes entre 1785 et 1805 . Nous étant penché depuis plusieurs années sur les systèmes prénominaux bas-normands et bretons, pour la période qui va de 1650 et $1850^{1}$, nous avons appris leur grande et permanente mobilité : modification de la longueur des prénoms complets variant avec le nombre des unités prénominales ${ }^{2}$, évolution sémantique du corpus, de sa hiérarchisation, etc. Partant de ce constat, il nous a semblé que devaient naître des questions de méthode quant à la recherche des "prénoms révolutionnaires" : il est probable que les systèmes prénominaux offrent d'autres signes éloquents de réaction, positive ou négative, à l'événement et que, dans ces conditions, s'en tenir aux seuls noms caractéristiques de l'anII risque de mutiler l'étude.

2 L'interrogation est particulièrement fondée chaque fois que les "prénoms révolutionnaires" ne s'imposent pas nettement, cas fréquent dans l'Ouest. Pour la ville de Rennes, c'est la sémantique du corpus et la longueur des prénoms qui retiendront principalement notre attention. Le dépouillement systématique des registres de catholicité puis de l'état civil a été entrepris pour la période 1785-1805, extrêmes incluses. Le dépouillement a été limité aux prénoms masculins dans le but d'alléger l'enquête ${ }^{3}$. Il a permis de rassembler 14144 prénoms complets.

Les prénoms révolutionnaires et les autres

3 À Rennes, le premier “prénom révolutionnaire” est en avril 1791: Pascal François Maurice Citoyen Français. Il faut attendre février 1793 pour qu'on dépasse une première fois la barre des 5\% des naissances, avec un Républicain et un Fargeau. Ensuite, il n'y a plus rien jusqu'en juillet. D'août 1793 à février 1794 (pluviôse anII), une reprise conduit à des niveaux plus modestes, entre 1,6 et 3,6\%, avec deux temps forts : en septembre (l'arrivée de Carrier étant saluée par un Sébastien Anne Carrier) et en nivôse anII, où les “prénoms révolutionnaires" représentent 9,7\% des naissances masculines, niveau 
presque record. Mais ce premier sommet est suivi d'un mois de pluviôse très faible, avec $1,9 \%$.

4 La plus belle période va de ventôse à fructidor an II, extrêmes inclus : pendant ces six mois, 33prénoms (soit 9,8\%) ont une unité "révolutionnaire". Ils se répartissent en quatre groupes à peu près équilibrés : valeurs politiques et morales (9références); héros contemporains et antiques (9également); calendrier révolutionnaire, avec ses évocations de la nature (8cas); enfin patriotisme (7références, en comprenant Regulus dans ce groupe).

La décrue est amorcée en vendémiaire anIII : encore 6\% des garçons nouveau-nés, mais au-delà on n'aura jamais plus de $5 \%$; après nivôse il n'y a plus rien, sauf un cas en prairial an II, un autre en messidor anIV (juillet 1796) et un dernier en brumaire anVII (septembre 1798). Dans cette phase tardive, les unités incriminées relèvent surtout du règne végétal, à travers le calendrier révolutionnaire (Jasmin, Romarin), et de la guerre (Ignace Canon), et nous éloignent du cœur politique des valeurs de l'an II.

6 Dans un département qui a donné relativement peu de "prénoms révolutionnaires" ${ }^{4}$, le bilan n'est pas négligeable, même si Dol et Saint-Malo font un peu mieux. Mais il est modeste. C'est moins qu'à Nantes et que dans d'autres villes de taille comparable ${ }^{5}$. Il correspond a priori au cas d'une ville classée comme "tiède" en l'anII : les autorités municipales ont d'abord appuyé l'action de Lanjuinais et des Brissotins, et participé au fédéralisme, sans s'y engager résolument, avant de se rallier à la Montagne. Carrier même dénonce cette tiédeur et appelle des renforts malouins. La Terreur prend à partir de janvier 1794 un visage modéré sous la direction de Esnuë-Lavallée et François, représentants en mission. Pour R. Dupuy, "la ville n'est [...] qu'apparemment sansculottisée".. La municipalité se réjouit finalement du 10 thermidor, et les principaux fédéralistes sont relâchés. La réaction thermidorienne après mai 1795 est elle-même modérée.

7 La démarche qui prévaut habituellement consiste, après avoir dégagé le noyau dur et indiscutable des "prénoms révolutionnaires", à déterminer la liste des unités prénominales qui peuvent entretenir avec l'épisode de l'anII des rapports plus complexes mais réels. La plupart du temps, on cherche des unités préexistantes mais revigorées en l'anII, ou intéressantes par leur polysémie : règne végétal, Antiquité, connotations morales, etc. Cette quête présente deux traits caractéristiques d'une part, elle est menée par discussion sur chaque unité prénominale, avec une marge d'incertitude variable ${ }^{7}$, à partir du ou des sens possibles et de la date d'apparition sur les registres; d'autre part, elle ignore les autres prénoms, confondus dans le même désintérêt : assez paradoxalement, peu d'études portent sur le répertoire chrétien au temps de la déchristianisation.

8 L'enquête de R.Bange ${ }^{8}$ montre à souhait la diversité des attitudes dans l'emploi de ce type de prénoms. Il n'est pas toujours possible d'en inférer immédiatement une réflexion sur les comportements politiques. En Normandie, à Falaise, nous avons eu l'occasion de rencontrer des fréquences de "prénoms révolutionnaires" encore plus basses (7,9\%, en comptant large, à l'été de l'an II) alors que l'engagement montagnard de la ville était plus net ${ }^{9}$.

9 La démarche que nous proposons ici ne consiste plus à partir des "prénoms révolutionnaires" en procédant ensuite par extension méthodique et raisonnée. Elle vise une double réinsertion: celle des "prénoms révolutionnaires" dans le reste du corpus ; celle des prénoms de l'époque révolutionnaire dans le temps plus long, celui du 
siècle quand cela est possible, en allant au moins au-delà de l'an II et même de la décennie $1789-1799^{10}$. Pour ce faire, une grille de lecture de l'ensemble du stock prénominal doit être établie, adaptée à son objet dans l'espace: Rennes, et dans le temps : 1785-1805. Il faut par exemple créer une catégorie "prénoms révolutionnaires", dans laquelle apparaîtra le noyau dur des références indiscutables. Au-delà du comptage de ce groupe, propre à la période, on observera avec attention le comportement de tous les types d'unités, laissées dans la catégorie qui est la leur avant la Révolution: les variations de leur fréquence peuvent prendre un sens politique fort, jusqu'à provoquer d'éventuels infléchissements sémantiques.

10 La constitution de cette grille rejette la réflexion unité par unité, précaution qui nous semble essentielle pour éviter les contresens, surtout lorsqu'on s'attache à des relations de sens trop univoques. On en donnera un exemple avec le cas très complexe de Louis. Ce nom peut certes renvoyer au roi de France. Mais cette référence n'explique ni l'inégalité de la répartition géographique, ni les différences chronologiques dans son destin, en France comme en Haute-Bretagne ${ }^{11}$. D'autres perspectives sont possibles: référence chrétienne d'abord, marquée par le roi du xiIIesiècle, éventuellement renouvelée au temps de la réforme catholique par saint Louis de Gonzague; référence politique ensuite, au sens le plus large, intégrant l'ensemble des monarques de l'Europe $\mathrm{du}$ XviIIesiècle : plus que la personne du roi, c'est l'ordre politique qui est en cause ${ }^{12}$. Enfin Louis peut jouer un autre rôle: celui d'unité fondamentale ${ }^{13}$ entrant de manière privilégiée dans la constitution de prénoms longs. Seule la comparaison avec d'autres unités prénominales, qui peuvent avoir une parenté de sens et ont une trajectoire historique proche (entrée dans le corpus ou augmentation de fréquence, point d'apogée, etc.), permet finalement une attribution. Les prénoms révolutionnaires en offrent un exemple particulier. Malgré la diversité de leurs répertoires, de la Rome antique aux fleurs des jardins, ils sont liés par leur sens politique et leur solidarité dans le temps. Généralement, c'est l'insertion dans le temps long qui est souvent éclairante.

11 La décision prise n'efface pas la complexité initiale. À Rennes, la place de Louis en tête $\mathrm{du}$ corpus depuis la première moitié du xviIIesiècle est déterminante, et en fait une fondamentale. Si le réseau de sens et de fonctions demeure, à commencer par la connotation politique, une réaction simple à la conjoncture révolutionnaire est désormais interdite. À la fin du siècle Louis a une fréquence de $4,8 \%$ par rapport à l'ensemble des unités prénominales et entre dans la composition de 9,4 à $10 \%$ des prénoms, selon les quinquennats. En 1793, cette proportion est abaissée à 7,6\%, le creux n'étant marqué que pour les deux premiers trimestres 5 et $6,3 \%$. Dès l'été 1793 , on retrouve des niveaux supérieurs à ceux des années 1785-1789. Non que les Rennais aient la mémoire courte, mais Louis n'est pas suffisamment marqué par la seule évocation du roi.

Il n'est pas possible de lever toutes les ambiguïtés et chaque grille doit assumer sa part d'arbitraire, sauf à renoncer à l'enquête elle-même. Il arrive que des classements dictés par la seule prudence soient indispensables : ainsi pour Guillaume et René dans cette étude ${ }^{14}$. Mais ces cas sont peu nombreux. En tout état de cause, il faut éviter d'attribuer à chacune des catégories créées une valeur exclusive de ses voisines: les "références historiques" associent Jules César et Napoléon, peu susceptibles de confusion avec le registre chrétien, à Casimir et à Charles, qui pourraient y appartenir, sans parler des prénoms byzantins, Léon ou Théophile. Leur présence commune dans cette rubrique renvoie à leur historicité d'une part, à leur rôle non exclusivement religieux d'autre 
part. Les références byzantines, pour chrétiennes qu'elles soient, ont des trajectoires parallèles à celles qui évoquent l'Antiquité grecque et romaine.

Référentiel des prénoms rennais

C'est à l'aide de cette grille que nous procéderons à l'examen du système prénominal rennais pendant la Révolution. Un premier tableau détaille les champs référentiels que nous avons retenus ${ }^{15}\left(\right.$ tableau $\left.^{\circ} 1\right)$.

Tableau $\mathrm{n}^{\circ} 1$ : Référentiel des unités prénominales rennaises (naissances légitimes 1785-1805) en $\%$

\begin{tabular}{|c|c|c|c|c|c|}
\hline & $1785-1789$ & $1790-1792$ & 1793-1795 & $1796-1800$ & $1801-1805$ \\
\hline Fondamentales & 62,3 & 64,6 & 61,0 & 60,8 & 62,4 \\
\hline Jean, Joseph, Marie, Pierre & 40,5 & 43,0 & 40,5 & 40,3 & 40,9 \\
\hline François, Julien, Louis & 20,1 & 19,9 & 19,1 & 19,2 & 19,7 \\
\hline Baptiste, Jean-Baptiste & 1,7 & 1,7 & 1,4 & 1,7 & 1,8 \\
\hline Références chrétiennes & 20,9 & 19,6 & 21,3 & 20,8 & 19,1 \\
\hline Anges, Évangiles & 8,3 & 8,2 & 9,2 & 9,2 & 8,0 \\
\hline Anges & 2,9 & 2,6 & 2,9 & 3,0 & 2,6 \\
\hline Apôtres & 3,7 & 4,3 & 4,1 & 4,2 & 3,6 \\
\hline Apôtres (époque de Paul) & 1,5 & 1,0 & 1,8 & 1,7 & 1,6 \\
\hline Divers & 0,2 & 0,3 & 0,4 & 0,3 & 0,2 \\
\hline Saints & 10,4 & 9,8 & 10,6 & 10,0 & 9,5 \\
\hline Saints légendaires & 3,8 & 3,5 & 3,4 & 3,3 & 3,1 \\
\hline Pères, docteurs de l'église & 1,2 & 1,2 & 1,6 & 1,5 & 1,2 \\
\hline Antiquité & 3,5 & 3,4 & 3,8 & 3,5 & 3,4 \\
\hline Moyen Age & 0,9 & 1,1 & 1,0 & 1,1 & 1,2 \\
\hline Depuis l'an Mil & 1,0 & 0,6 & 0,8 & 0,6 & 0,6 \\
\hline Réf. chrétiennes diverses & 2,1 & 1,6 & 1,5 & 1,6 & 1,6 \\
\hline Noms bibliques & 0,2 & 0,1 & 0,2 & 0,3 & 0,1 \\
\hline Divers & 1,9 & 1,5 & 1,3 & 1,3 & 1,5 \\
\hline
\end{tabular}




\begin{tabular}{|c|c|c|c|c|c|}
\hline Réf. historiques, morales & 7,7 & 8,5 & 11,0 & 11,4 & 12,2 \\
\hline Mythologie, théâtre & 1,5 & 1,4 & 1,6 & 1,7 & 1,9 \\
\hline Antiquité & 0,5 & 0,7 & 0,8 & 0,8 & 1,0 \\
\hline Moyen Age, époque moderne & 1,0 & 0,7 & 0,8 & 0,7 & 0,9 \\
\hline Références historiques & 4,7 & 5,1 & 7,4 & 7,3 & 9,1 \\
\hline Antiquité & 1,3 & 1,2 & 2,1 & 1,7 & 2,3 \\
\hline Monde byzantin & 0,6 & 0,4 & 0,7 & 1,0 & 1,0 \\
\hline Moyen Age & 0,4 & 0,5 & 0,5 & 0,8 & 0,6 \\
\hline Époque contemporaine & 2,4 & 3,0 & 2,5 & 3,8 & 4,0 \\
\hline Prénoms révolutionnaires & 0,0 & 0,0 & 1,3 & 0,0 & 0,0 \\
\hline Napoléon & 0,0 & 0,0 & 0,0 & 0,0 & 0,2 \\
\hline Références morales & 1,5 & 2,0 & 2,0 & 2,4 & 2,2 \\
\hline Noms de saints & 0,9 & 1,2 & 1,0 & 1,3 & 1,4 \\
\hline Autres noms & 0,6 & 0,8 & 1,0 & 1,1 & 0,8 \\
\hline Références bretonnes & 2,0 & 1,6 & 1,2 & 1,3 & 1,2 \\
\hline Province & 0,2 & 0,1 & 0,2 & 0,1 & 0,2 \\
\hline Saints bretons & 1,8 & 1,5 & 1,0 & 1,2 & 1,0 \\
\hline Prénoms féminins & 2,4 & 2,0 & 1,7 & 1,6 & 1,5 \\
\hline Divers & 4,9 & 3,6 & 4,1 & 3,9 & 3,5 \\
\hline
\end{tabular}

14 La presque totalité des unités prénominales relève de trois ensembles, dont la fréquence cumulée dépasse 95\%. Le premier réunit les unités de tête du corpus, ou fondamentales. Ce sont toutes des références chrétiennes, avec cependant un rapport inégal à la personne du Christ et au cœur de la Révélation : Marie en est sans doute plus proche que François ou Louis. Surtout ces unités ont pour caractéristique d'être choisies pour allonger les prénoms, jouant le rôle de "briques élémentaires" et leur fréquence s'explique en partie par cet attrait du prénom long. Les références chrétiennes forment le deuxième ensemble: souvent inscrites sur les calendriers diocésains, elles ont généralement constitué le fonds prénominal usuel depuis la fin du Moyen Âge. Le troisième groupe, celui des références historiques et morales, porte encore des 
significations chrétiennes mais le lien avec la religion est moins certain en raison de références à d'autres domaines: histoire ou morale pour l'essentiel. C'est naturellement dans cette catégorie d'unités que les "prénoms révolutionnaires" trouvent leur place. La division des vingt et une années étudiées en périodes de trois ou cinq ans correspondant grossièrement au développement historique montre avant tout la grande stabilité du référentiel rennais.

15 À considérer les trois grandes masses qui viennent d'être nommées, aucune rupture ne se dessine clairement, correspondant par exemple à la phase radicale de l'anII. On relèvera pourtant le progrès des références historiques et morales à partir de la période 1793-1795. Les "prénoms révolutionnaires" ne comptent que très partiellement dans cet accroissement de fréquence: leur influence est modeste en elle-même (d'autant qu'il s'agit d'un calcul effectué par rapport au total des unités prénominales, non par rapport au total des enfants) et limitée dans le temps. Ce succès très relatif des prénoms historiques ne peut être imputé à aucune sous-catégorie en particulier. Toutes les rubriques ou presque connaissent une légère appréciation de leur fréquence. De même, aucune autre catégorie en particulier n'a à souffrir de cette appréciation. Les références chrétiennes traversent la Révolution sans marquer de recul. Leur stabilité d'ensemble, comme celle des sous-catégories constitutives est indiscutable.

Qu'est-ce à dire ? Rennes passe-t-elle à côté de la déchristianisation de l'an II ou bien nos instruments de mesure sont-ils trop grossiers? Détaillant chacune des trois années 1793, 1794 et 1795, on fait apparaître des signes plus nets de réaction à la conjoncture politique :

Tableau n² : Les unités prénominales de 1793 à 1795 (en \%)

\begin{tabular}{|l|l|l|l|}
\hline & 1793 & 1794 & 1795 \\
\hline \hline Fondamentales & 63,1 & 58,1 & 61 \\
\hline \hline Références chrétiennes & 20,8 & 20,5 & 21,3 \\
\hline \hline Anges, Évangiles & 8,1 & 8.8 & 9,8 \\
\hline \hline Saints & 10,8 & 10,4 & 10,1 \\
\hline \hline Divers & 1,9 & 1,3 & 1,4 \\
\hline \hline Références historiques et morales & 8,5 & 14,2 & 11,0 \\
\hline \hline Mythologie, théâtre & 0,9 & 2,0 & 1,7 \\
\hline \hline Histoire (sauf "prénoms révolutionnaires") & 5,3 & 4,1 & 6,9 \\
\hline \hline "Prénoms révolutionnaires" & 0,7 & 4,5 & 0,2 \\
\hline \hline Références morales & 1,6 & 2,6 & 2,2 \\
\hline \hline Autres & 7,6 & 7,0 & 6,6 \\
\hline
\end{tabular}




\begin{tabular}{|l|l|l|l|l|l|l|l|l|}
\hline Total & 1285 & 919 & 1163 \\
\hline
\end{tabular}

171793 se présente comme une année banale, les différences avec la période précédente, 1790-1792, sont infimes et statistiquement sans valeur. La rupture - si toutefois ce mot n'est pas trop fort - est à chercher en 1794: 4,5\% d'unités prénominales "révolutionnaires" et un bloc de références historiques et morales hissé à 14,2\%. L'an II laisse une trace modeste mais indiscutable dans le système prénominal rennais. Elle semble s'effacer l'année suivante, en 1795. C'est donc sur l'année 1794 que doit porter l'examen de détail.

18 Les “prénoms révolutionnaires" n'ont pas mordu sur le stock des références chrétiennes. À scruter le détail des évolutions, on n'observe même pas la trace d'un renouvellement interne ${ }^{16}$. C'est à la tête du corpus, dans le groupe des fondamentales, qu'on trouvera les principales victimes des "prénoms révolutionnaires". Avec 58,1\%, il fait le score le plus faible des vingt et uneannées de l'étude. Le détail montre que c'est surtout le premier ensemble qui régresse : Jean, Pierre, Marie, et Joseph, qui relèvent tous du fonds chrétien. Ce serait donc à travers ses références les plus banales que ce registre serait touché.

Quelques signes particulièrement sensibles montrent que les références chrétiennes, dans leur globalité, ont bien été atteintes mais qu'elles ne l'ont été que superficiellement.

Tableau n³ : Évolution de quelques références chrétiennes (en \%)

\begin{tabular}{|l|l|l|l|}
\hline & Fréquence de Marie & Fréquence de Baptiste & Fréquence des associations Marie-Joseph ${ }^{17}$ \\
\hline \hline $1785-1789$ & 21 & 3,3 & 9,7 \\
\hline \hline $1790-1792$ & 25,6 & 3,5 & 9,6 \\
\hline \hline 1793 & 24,9 & 2,5 & 10,3 \\
\hline \hline 1794 & 15 & 2,3 & 11 \\
\hline \hline 1795 & 20,5 & 2,5 & 6,4 \\
\hline \hline $1796-1800$ & 24,7 & 3,5 & 10,2 \\
\hline \hline $1801-1805$ & 25,8 & 3,6 & 9,9 \\
\hline
\end{tabular}

Une des caractéristiques majeures du système prénominal de Haute-Bretagne est l'usage courant pour les garçons d'inclure Marie dans le nom complet il en est ainsi depuis que le prénom a commencé de s'allonger ${ }^{18}$. On observe bien en 1794 une fréquence anormalement faible mais le recul est mesuré : la fréquence pour les trois années 1793-1795 - 20,3\% - est comparable à celle de la période 1785-1789 : 21\%. Il serait d'ailleurs dangereux, pour expliquer le phénomène, de s'en tenir à une imputation d'ordre strictement religieux. Ce recul observé en l'anII pourrait avoir été favorisé par le raccourcissement des prénoms complets, à la même époque, sur lequel 
nous reviendrons plus loin de même qu'au XviIIesiècle toutes les unités fondamentales ont été poussées en avant par la mode des prénoms longs, le recul de ces derniers aurait entraîné celui de ces unités. L'association de Marie et de Joseph, qui amplifie le caractère chrétien de la référence, n'est pas affectée par les troubles de la période, sauf pour l'année... 1795 !

Baptiste, précédé ou non de Jean, se tient pendant troisans sur un palier moins élevé qu'avant 1792, mais la différence est médiocre et disparaît après 1795. On ferait encore des observations analogues avec les noms qui évoquent les temps forts du calendrier chrétien: Toussaint, Pascal, Noël, Emmanuel, qui constituent l'essentiel des références chrétiennes diverses. On n'observe pas pour eux de repli significatif entre 1793 et 1795. Toussaint, la plus importante de ces unités, passe d'un palier situé à 1,6\% depuis 1785 , à $0,8 \%$ en 1794, mais la reprise est commencée dès 1795 ; sous Bonaparte, elle retrouve les niveaux qu'elle avait sous Louis XVI. Il y a plus: le lien patent qui existe entre le calendrier et l'octroi de ces unités à un nouveau-né est maintenu à travers la période : entre 1785 et 1792, 32,3\% des 127 Toussaint et Noël sont donnés dans les semaines entourant la date de référence de 1793 à 1795, la proportion est de $31 \%$ pour 29 unités, de $25 \%$ de 1796 à 1805 pour 127 autres unités ${ }^{19}$.

Le référentiel chrétien ne montre pas d'autre signe d'affaiblissement. En 1794, il n'est touché que de façon marginale et, dès 1795 , il se retrouve identique à lui-même. En sens inverse, un nom traduit en 1799 (ventôse anVII) un attachement chrétien fort : Jean Dieu Pierre, sans doute une référence à saint Jean de Dieu, mais ce cas est unique et ne traduit pas non plus une quelconque "contre-offensive" des noms chrétiens postérieure à la crise de l'anII.

23 Les renvois à l'histoire ne progressent pas en 1794, bien au contraire, glissant de 5,3 à $4,1 \%$. En revanche, le repli des "prénoms révolutionnaires" l'année suivante se solde par un bond à $6,9 \%$ de ces références : tout se passe comme si elles étaient puisées sinon au même fonds, du moins dans des registres très voisins. Les preneurs de prénoms révolutionnaires se recruteraient essentiellement dans les catégories sociales qui, hors le contexte de l'anII, choisiraient des évocations de l'Antiquité, par exemple, pour nommer leurs enfants. Il n'en va pas nécessairement de même partout mais, si cette hypothèse est fondée, on pourrait parler de radicalisation des éléments "avancés" sans gain véritable sur le reste de la population. D’un point de vue méthodologique, on relèvera que l'argument d'un regain de fréquence en l'anII, qui sert à désigner des proches des "prénoms révolutionnaires", doit être manié avec précaution.

24 Si pour l'essentiel, les "prénoms révolutionnaires" disparaissent après 1794 ou 1795, le bloc références historiques et morales, lui, consolide les gains qu'il a réalisés, essentiellement par le biais des renvois au théâtre et à la mythologie, à l'Antiquité et au monde contemporain. Il faudrait cependant un plus grand recul pour apprécier l'importance de ce changement et son caractère durable.

Étude sociale du référentiel

Les registres de l'état civil permettent d'apporter des compléments nécessaires les informations d'ordre socio-professionnel étant plus nombreuses que sur les registres paroissiaux, particulièrement à partir de 1794, le détail des comportements par groupe social est rendu possible. Il montre effectivement des différences importantes, qu'on saisit dès avant la Révolution, en se fondant sur les quelques indications d'honorabilité que les registres paroissiaux fournissent avec les avant-noms. 
Tableau $n^{\circ} 4$ : Organisation sociale du système de références, 1785-1792 (en \%)

\begin{tabular}{|c|c|c|c|c|c|c|c|}
\hline & 1 & 2 & 3 & 4 & 5 & 6 & 7 \\
\hline Fondamentales & 31,7 & 50 & 55,1 & 59,7 & 64,3 & 64,2 & 62,3 \\
\hline Références chrétiennes & 21,5 & 20,3 & 19 & 20,8 & 20 & 20,3 & 22,6 \\
\hline Anges, Évangiles & 8,9 & 8,5 & 8,5 & 6,4 & 10 & 8,3 & 10,4 \\
\hline Saints & 12,7 & 10,4 & 8,9 & 13,2 & 9,4 & 10 & 9,8 \\
\hline Divers & 2,5 & 1,4 & 1,6 & 1,2 & 0,6 & 2 & 2,5 \\
\hline $\begin{array}{l}\text { Références historiques et } \\
\text { morales }\end{array}$ & 36,5 & 16,6 & 16,3 & 12,0 & 5,2 & 7,2 & 7,6 \\
\hline Mythologie, théâtre & 3,8 & 2,2 & 1,0 & 1,0 & 1,0 & 1,8 & 1,3 \\
\hline Histoire & 24,0 & 9,4 & 11,1 & 8,3 & 3,0 & 4,2 & 4,6 \\
\hline Références morales & 8,7 & 5 & 4,2 & 2,7 & 1,2 & 1,5 & 1,7 \\
\hline Autres & 7,6 & 12,2 & 6,8 & 7,4 & 10,9 & 8,4 & 7,6 \\
\hline Total & 79 & 222 & 189 & 560 & 322 & 7863 & 1348 \\
\hline
\end{tabular}

1 : Hauts et puissants seigneurs ; 2 : Nobles maîtres ; $\mathbf{3}$ : Nobles hommes ; 4 : Sieurs ; 5 :Honnêtes hommes ; 6 : Sans avant-nom ; 7 : Pères inconnus.

Encore une fois, le groupe des références chrétiennes frappe par sa permanence, en bloc comme en détail. Il offre aux Rennais un répertoire d'ailleurs très riche, constituant à lui seul près de la moitié du catalogue en usage. Les noms qui évoquent les anges et les évangiles, les saints légendaires ou miraculeux, sont encore peu nombreux mais ils rassemblent beaucoup de suffrages; en revanche les saints dispersés dans les différentes époques de l'histoire chrétienne n'apparaissent que rarement (les fréquences sont souvent inférieures à $0,1 \%$ ) mais leur nombre considérable donne à la catégorie toute son l'importance. La dispersion des noms chrétiens n'empêche pas le maintien d'une fréquence globale à un niveau presque constant, dans le temps comme entre les différentes catégories sociales.

Du fait de cette stabilité, la distinction sociale se joue pour l'essentiel dans la balance entre fondamentales et références historiques et morales. Le comportement de la masse des Rennais est défini par les trois dernières colonnes, l'expression "honnête homme" s'avérant n'être ici que le médiocre embellissement d'une situation sociale banale. À elles trois, elles regroupent plus de $90 \%$ des références identifiées. Les fondamentales, qui ne sont que huit, ont une énorme fréquence cumulée : presque les deux tiers du stock. Il n'y a plus de place pour les évocations historiques ou morales que pour $4 \%$ environ des références prénominales. 
28 Au-dessus de ces catégories sociales, et malgré l'étroitesse des échantillons, une cohérence se dessine : la part des fondamentales est d'autant plus faible qu'on monte dans la hiérarchie sociale, la noblesse fournissant un exemple extrême ${ }^{20}$. Le plus intéressant est que cet amenuisement profite aux références historiques et morales, même si d'autres groupes de prénoms ont bénéficié d'initiatives spectaculaires. Ainsi, pour les références bretonnes, voit-on en janvier 1785 le président de l'ordre de la noblesse aux États de Bretagne appeler son fils François Bretagne Urbain Marie Nicolas Maurice. L'exemple est imité quelques années plus tard par le maire de Rennes, Talhouët, qui le 21août 1792 nomme son fils Rennes Éleuthère Félix René Joseph. Mais ces références à la province et à la ville ne sont guère que des apax et c'est bien au sein des groupes qui évoquent l'Europe du temps ou l'Antiquité, ou qui jouent sur l'ambiguïté entre noms chrétiens et qualités morales, qu'on trouve les développements les plus importants.

Ce ne sont pas tant d'ailleurs les souvenirs du théâtre et de la mythologie, peu surprenants dans ces élites cultivées, qui font la différence. Pas plus que dans le registre historique le Moyen Âge occidental ou byzantin ne joue un rôle discriminant efficace. La fréquence des références à cet univers, presque ignoré par la masse, atteint 6,3\% dans la noblesse mais seulement 2,2\% chez les "nobles maîtres". En revanche, les évocations de l'Antiquité grecque et surtout romaine [Auguste et Alexandre surtout ${ }^{21}$, celles qui désignent la France et l'Europe des contemporains (Charles, Henri, Frédéric et Adolphe), et les prénoms qui renvoient à une qualité morale (Aimé, Félix, etc.) ont un poids remarquable. La fréquence cumulée de ces rubriques, hypertrophiée pour la noblesse (26,6\%), est de $12,2 \%$ pour les "nobles maîtres" et 13,7\% les "nobles hommes", alors qu'elle est seulement de $5,2 \%$ pour les nouveau-nés dont les pères n'ont pas d'avant-nom. Cette élite, qui associe déjà, avant la Révolution, choix du prénom et souci d'une réflexion partiellement laïcisée, est préparée au prénom-programme. Ainsi s'expliquent les ouvertures à des unités originales comme Rennes ou Bretagne, et, plus tard, aux "prénoms révolutionnaires".

Tableau $n^{\circ} 5$ : Organisation sociale du système de références, 1793-1805 (en \%)

\begin{tabular}{|l|l|l|l|l|l|l|l|}
\hline 1.1793 à 1795 & 1 & 2 & 3 & 4 & 5 & 6 & 7 \\
\hline \hline Fondamentales & 51,2 & 45,7 & 51,3 & 63,4 & 68,7 & 65,3 & 60,4 \\
\hline \hline Références chrétiennes & 16,8 & 19,7 & 27,8 & 22 & 16,5 & 20,6 & 15,6 \\
\hline \hline Anges, Évangiles & 8,4 & 10,4 & 9,9 & 9,8 & 9 & 8,6 & 6,4 \\
\hline \hline Saints & 6 & 7,8 & 15,6 & 11 & 6,6 & 11 & 7,6 \\
\hline \hline Divers & 2,4 & 1,5 & 2,3 & 1,2 & 0,9 & 1 \\
\hline \hline $\begin{array}{l}\text { Références historiques } \\
\text { et morales }\end{array}$ & 23,8 & 27,9 & 14,4 & 7,2 & 8,4 & 7,9 \\
\hline \hline Mythologie, théâtre & 1,2 & 4,1 & 2,6 & 1,2 & 0,9 & 2 \\
\hline \hline Histoire & 12,0 & 13,7 & 6,9 & 4,4 & 5,4 & 4,5 \\
\hline
\end{tabular}




\begin{tabular}{|c|c|c|c|c|c|c|c|}
\hline $\begin{array}{l}\text { "Prénoms } \\
\text { révolutionnaires" }\end{array}$ & 6 & 5,2 & 1,9 & 0,7 & 0,9 & 0,7 & 5,2 \\
\hline Références morales & 3,6 & 4,9 & 3 & 1,6 & 2,1 & 1,4 & 3,2 \\
\hline Autres & 9,6 & 6,4 & 6,5 & 7,2 & 6,3 & 5,9 & 6,8 \\
\hline Total & 84 & 269 & 263 & 743 & 333 & 288 & 250 \\
\hline 2. 1796 à 1805 & 1 & 2 & 3 & 4 & 5 & 6 & 7 \\
\hline Fondamentales & 48,5 & 47,8 & 58,1 & 63,2 & 71,5 & 66,7 & 61,3 \\
\hline Références chrétiennes & 20,7 & 20,5 & 21,9 & 20,5 & 16,6 & 20,2 & 20,9 \\
\hline Anges, Évangiles & 8,2 & 7,7 & 8,9 & 9 & 6,6 & 10,2 & 9,2 \\
\hline Saints & 10,9 & 11,1 & 10,7 & 9,8 & 8,8 & 8,8 & 9,7 \\
\hline Divers & 1,6 & 1,8 & 2,2 & 1,7 & 0,9 & 1,3 & 2,1 \\
\hline $\begin{array}{l}\text { Références historiques } \\
\text { et morales }\end{array}$ & 23,1 & 27,0 & 13,5 & 9,0 & 5,6 & 6,2 & 11,2 \\
\hline Mythologie, théâtre & 3,4 & 4 & 2,6 & 1,2 & 0,8 & 0,9 & 1,3 \\
\hline Histoire & 15,8 & 17,8 & 8,9 & 5,9 & 3,7 & 4,0 & 7,4 \\
\hline $\begin{array}{l}\text { "Prénoms } \\
\text { révolutionnaires" }\end{array}$ & 0 & 0,5 & 0,1 & 0 & 0 & 0 & 0 \\
\hline Références morales & 3,8 & 4,7 & 2,8 & 1,9 & 1 & 1,3 & 2,3 \\
\hline Autres & 6,2 & 4,7 & 6,3 & 7,2 & 6,5 & 6,8 & 6,5 \\
\hline Total & 633 & 1307 & 1296 & 4760 & 1892 & 1374 & 1861 \\
\hline
\end{tabular}

1: Inactifs; 2: Professions libérales, administrateurs, officiers militaires; 3 : Marchands, négociants ; 4 : Patrons et salariés de l'artisanat ; 5 : Laboureurs ; 6 : Salariés ; 7 : Pères inconnus.

De fait, à Rennes, ce sont les élites qui vont promouvoir les prénoms nouveaux. Il suffit pour s'en convaincre de considérer le tableau $n^{\circ} 5$. La nomenclature socioprofessionnelle des registres de l'état civil n'étant plus celle des registres paroissiaux, les groupes constitués ici ne sont pas immédiatement comparables à ceux qui avaient servi plus haut. Une hiérarchie sociale apparaît néanmoins, qui reproduit les comportements prénominaux déjà repérés.

31 Pour les artisans, laboureurs et salariés, la part des fondamentales est énorme, soulignant la grande monotonie des prénoms donnés aux enfants. Le rapport entre 
références chrétiennes et références historiques et morales n'a guère évolué par rapport à ce que laissait entrevoir le tableau $\mathrm{n}^{\circ} 4$ pour les enfants dont les pères n'avaient pas d'avant-nom. Dans ces groupes, les "prénoms révolutionnaires" sont une infime minorité.

Les marchands et les négociants, les entrepreneurs fournissent une sorte de transition : les fondamentales sont moins importantes, mais c'est en partie au profit des références chrétiennes. Situation intermédiaire aussi, mais différente, pour les nouveau-nés venus d'unions illégitimes ${ }^{22}$ : quelques "prénoms révolutionnaires" tranchent sur la masse par la netteté de leur engagement (Dix-Août, Scaevola, Barra). Le fait n'est pas propre à Rennes et se retrouve par exemple dans les Côtes-du-Nord ${ }^{23}$. En sus du rôle des officiers de santé et des sages-femmes qui accouchent ces jeunes mères souvent venues des campagnes environnantes, et viennent déclarer l'enfant, ou de celui des témoins, employés de l'administration ou militaires, il faudrait mesurer le nombre des unions non encore légalisées de soldats et d'officiers qui pour la plupart sont aux armées lorsque naît l'enfant. L'état civil offre quelques exemples de légalisation tardive de ces unions. Des patronymes à consonance étrangère à la province - noms alsaciens ou lorrains par exemple - ou des indications sur le lieu de naissance de la mère incitent encore à des explications de ce genre. Or nous montrerons bientôt que c'est dans l'armée que figurent les éléments les plus favorables à l'essor de prénoms nouveaux.

Les deux premières colonnes distinguent le reste des élites. Si la noblesse ne sert plus de référence comme par le passé, la part des fondamentales est néanmoins très basse. Ce sont les évocations de l'histoire et les "prénoms révolutionnaires" qui apparaissent comme les signes distinctifs de ces catégories, qui rassemblent les individus placés aux postes de responsabilité: magistrats et avocats, receveurs et employés des contributions directes, médecins et professeurs de collège, officiers de l'armée et administrateurs des hôpitaux ou de l'intendance militaires, etc., à quoi il faut ajouter les rentiers. C'est dans ce monde-là que l'on trouve les évocations souvent érudites de l'Antiquité, des sciences et de la pensée contemporaine (Érasme, Euler, Franklin); c'est dans ce milieu aussi qu'apparaissent les "prénoms révolutionnaires"; c'est encore lui qui fournit en 1805 le plus de petits Napoléon.

Il y a lieu de préciser l'apport de cette catégorie. Trois sous-ensembles doivent être à leur tour distingués. Le premier concerne les officiers et sous-officiers de l'armée, les administrateurs des hôpitaux et de l'intendance militaires. En 1794 et 1795, ils déclarent à l'état civil 49nouveau-nés : les références historiques et morales représentent en 1793-1795 35,1\% du stock. Trois enfants seulement ont Marie dans leur prénom. En revanche, on relève cinq "prénoms révolutionnaires" (Viala, Guillaume Tell, Gracchus, Scaevola, Fleurus), auxquels il faut ajouter des noms de héros antiques (Marius, Paul Émile, Aristide, Timoléon), et des noms à connotation morale rares : Sincère. Aucune catégorie n'a autant fait pour le prénom révolutionnaire, est-on tenté de dire, d'autant que ces noms sont sans ambiguïté, au cœur des valeurs politiques de la Révolution. Le fait d'appartenir à l'armée ou aux structures administratives, qui suppose en 1794 un engagement politique clair, ne suffit pas pour expliquer l'abondance de ces prénoms. Ils restent principalement la marque des cadres dirigeants, à la différence des noms des enfants de soldats et d'ouvriers des arsenaux d'une part, des employés des hôpitaux ou de l'intendance d'autre part: soit les catégories placées directement sous les ordres de la première. Il se trouve que le nombre de nouveau-nés déclarés est comparable 48 pour les soldats, 47 pour les employés. Les premiers offrent deux "prénoms 
révolutionnaires" seulement (Brutus, Égalité) pour huit Marie et aucune référence à l'Antiquité ; les seconds trois prénoms révolutionnaires (deux Raison et un Jasmin) pour dix Marie et cinq références à l'Antiquité (dont trois Auguste).

Reste que militaires et personnel des administrations ont un degré d'enracinement dans la ville inégal. Officiers et sous-officiers ne sont pas toujours originaires de Rennes, ni même de Bretagne. Ils n'y font que passer au rythme des opérations militaires ; épouses et enfants déclarés à l'état civil n'y resteront pas nécessairement non plus. Le médiocre nombre de prénoms avec Marie trouve peut-être là son explication. Le personnel responsable des hôpitaux est sans doute plus stable mais il faudrait mieux le connaître, tout comme celui des administrations civiles. Juges des tribunaux, avocats, médecins ont des liens avec la société locale sans doute plus forts que les militaires. Leur comportement est plus nuancé. Pour 36enfants, un seul Regulus mais douze Marie et surtout des évocations de l'Antiquité qui ne surprennent pas dans ces milieux cultivés: Aristide, Paul-Émile, Auguste et plusieurs Hyppolite, auxquels s'ajoutent Félix, Désiré. C'est dans ce registre prudent qu'apparaissent aussi les noms de 44 enfants déclarés par des pères qui ne donnent aucune indication socio-professionnelle: caractéristique qui sera plus tard, sous le Directoire et plus encore le Consulat, celle de rentiers porteurs de noms à tiroirs, ou même d'aristocrates facilement reconnaissables. Sont-ce déjà des rentiers en 1794, ce qui permettrait de les rapprocher des précédents, ou faut-il se borner à invoquer les lacunes du document? Si l'on relève quelques prénoms révolutionnaires indiscutables (Coq, Thermidor et deux Regulus dominant un Marat), 1795 voit dans ce groupe, en même temps que la disparition de ces noms, le retour en force des Marie.

Doit-on conclure que le système prénominal rennais n'a été qu'égratigné par la Révolution, en soulignant qu'il ne l'a été que pour des catégories elles-mêmes engagées politiquement dans le processus révolutionnaire, ce qui serait encore une manière de marquer la résistance des habitants au changement? C'est précisément ce type de conclusion qu'il faut fuir tout pendant que le système prénominal n'a pas été examiné complètement.

La longueur des prénoms pendant la Révolution.

L'allongement du prénom a été la première rupture avec le système prénominal classique légué par la fin du Moyen Âge. Observé partout à des dates différentes, ce phénomène est commencé à Rennes à la fin du xviresiècle. À la fin du siècle suivant, toutes les catégories ne se sont pas encore donné de prénoms longs. Nous avions déjà observé sur le site de Falaise le raccourcissement brutal, et éphémère, des prénoms complets en l'an $\mathrm{II}^{24}$. Rennes fournit l'occasion d'un autre test sur un échantillon plus grand, avec une nomenclature socioprofessionnelle plus complète. Le graphique donne la fréquence relative des prénoms simples, à une seule unité, trimestre par trimestre.

Alors que les prénoms simples représentaient 20 à $25 \%$ des noms d'enfants nés d'unions légitimes depuis 1785, la courbe est portée brutalement à 35 puis $45 \%$ au début de 1794 . L'amplitude de la variation est très inhabituelle et il faut remonter plusieurs décennies en arrière pour trouver pareille proportion de prénoms simples. Cette poussée des noms formés d'une seule unité correspond au recul de ceux qui en comportent trois ou plus, la statistique ne faisant pas apparaitre de variation majeure pour les noms à deux unités. On peut alors imaginer, plus qu'un passage brutal de trois à une unité, une série de glissements de trois à deux, et de deux à une. 


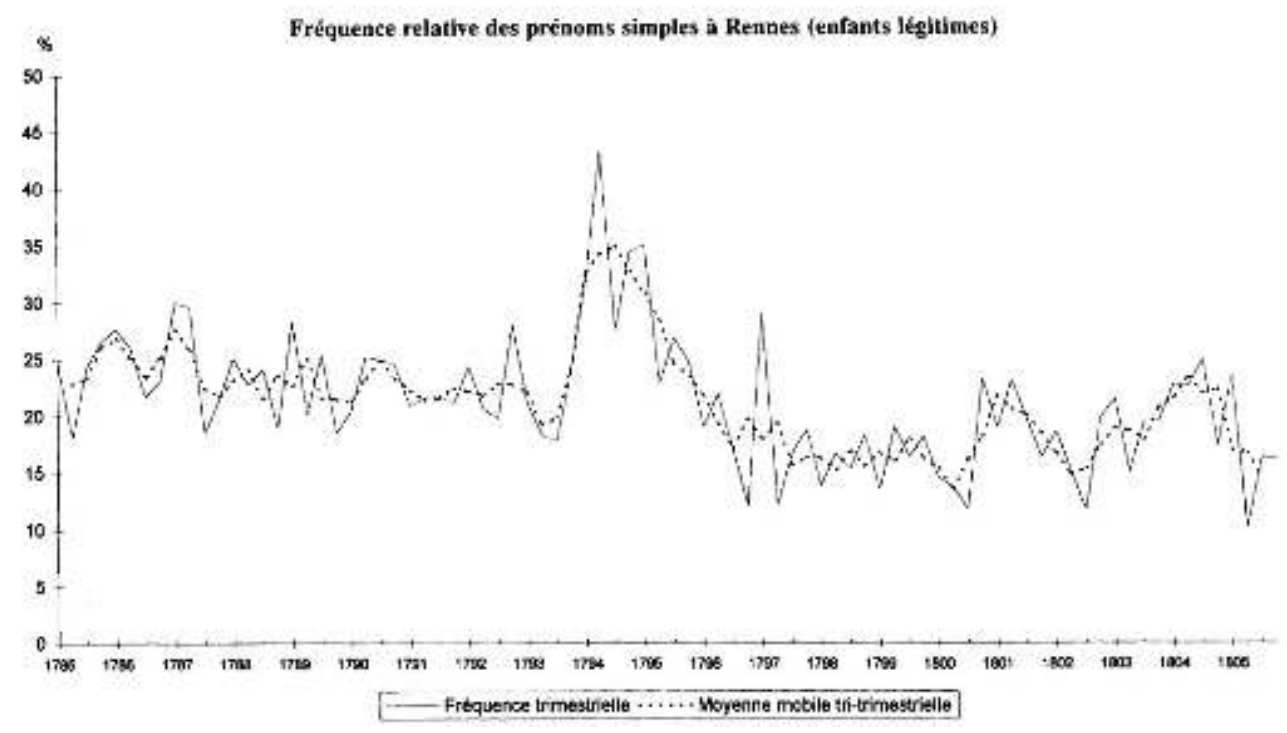

Il n'est pas inutile d'entrer dans le détail du phénomène, mois par mois, entre juillet 1793 et juin 1795 (prairial anIII). Jusqu'au début de 1794, rien d'inhabituel ne s'est encore produit; en nivôse anII, on compte parmi les naissances légitimes $20,6 \%$ de prénoms simples et $18,1 \%$ de prénoms à trois unités ou plus : ce qui entre dans les fourchettes de la période précédente. Brutalement, en pluviôse anII, la proportion de prénoms longs tombe au dessous de $5 \%$, celle des prénoms courts étant portée à $47,9 \%$; ces valeurs sont maintenues jusqu'en prairial, avec un maximum de prénoms courts en floréal : 57,1\%! Les deux premiers mois de l'été voient une très sensible décrue, suivie d'une reprise en fructidor et vendémiaire ( $45,7 \%$, et $4,3 \%$ pour les prénoms longs). Passé ce cap, les prénoms à trois unités retrouvent définitivement une fréquence habituelle, autour de $18 \%$, ceux qui n'ont qu'une unité passant encore par des valeurs élevées en nivôse puis en ventôse de l'anIII. Au-delà, la proportion de prénoms courts tombe à des niveaux inférieurs à ceux d'avant 1792, qui seront conservés tout au long $\mathrm{du}$ Directoire; on n'enregistre plus que quelques écarts, en creux ou en pointe, qui laissent une moyenne assez stable. Cette chronologie est celle des "prénoms révolutionnaires”, qui n'ont à Rennes de fréquence significative qu'au premier semestre de 1794.

Amplitude et chronologie appellent l'attention sur cette évolution; mais avant de s'interroger sur le sens qu'il faut lui donner, il faut tenter d'en décrire les contours sociaux. La hiérarchie antérieure à 1793 s'établit comme suit :

Tableau n 6 : Longueur des prénoms (1785-1792)

\begin{tabular}{|l|l|l|l||l|l|l|l|}
\hline & 1 & 2 & 3 & 4 & 5 & 6 & 7 \\
\hline \hline une unité & 12,9 & 8,1 & 5,2 & 7,5 & 13,1 & 25,4 & 36,2 \\
\hline \hline deux unités & 38,7 & 36,3 & 45,5 & 51,9 & 59,5 & 55,4 & 52,4 \\
\hline \hline trois unités & 29 & 43,1 & 39 & 37,8 & 27,5 & 17,4 & 10,9 \\
\hline \hline quatre unités et plus & 19,4 & 12,5 & 7,8 & 2,9 & 0 & 1,9 & 0,5 \\
\hline
\end{tabular}




\begin{tabular}{|c|c|c|c|c|c|c|c|}
\hline total & 31 & 160 & 77 & 241 & 153 & 4088 & 781 \\
\hline \multicolumn{8}{|c|}{$\begin{array}{l}1: \text { Hauts et puissants seigneurs ; } 2: \text { Nobles maîtres ; } \mathbf{3}: \text { Nobles hommes ; } 4 \text { : Sieurs ; } \\
5 \text { : Honnêtes hommes ; } 6 \text { : Sans avant-nom ; } 7 \text { : Pères inconnus. }\end{array}$} \\
\hline
\end{tabular}

La norme à Rennes à la fin du xvinesiècle est le prénom à deux unités. Pour autant, le tableau montre que les écarts sont importants. Les élites portent des prénoms longs ; historiquement, elles ont été les premières à procéder à cet allongement et il semble même que l'aristocratie ait précédé tout le monde ${ }^{25}$. Entre 1785 et 1792, autant qu'on puisse en juger sur ces échantillons, les élites roturières l'ont rattrapée, voire dépassée. Les prénoms les plus longs (quatre unités ou plus) sont pourtant ceux de la noblesse, "nobles maîtres" ou "nobles hommes" s'arrêtant plus souvent à trois.

Inversement, dans les milieux populaires, le prénom simple suffit encore au quart des enfants. La proportion est plus élevée pour les enfants nés d'unions illégitimes : peutêtre parce que le choix du nom, qui prend partiellement la forme d'une transmission du nom des parents naturels, ne peut plus s'effectuer ici de manière banale ; ou peut-être, et plus simplement, parce que ces naissances qu'on cherche souvent à masquer, appellent un choix plus modeste qu'un prénom long contredirait.

Tableau $n^{\circ} 7$ : Longueur des prénoms (1793-1795)

\begin{tabular}{|l||l||l|l|l||l|l|l|}
\hline & 1 & 2 & 3 & 4 & 5 & 6 & 7 \\
\hline \hline une unité & 27,3 & 24,4 & 28,4 & 28,5 & 42,3 & 32,3 & 40 \\
\hline \hline deux unités & 56,8 & 47,7 & 48,5 & 60,4 & 48,8 & 61,7 & 46,9 \\
\hline \hline trois unités & 13,6 & 27,3 & 20,9 & 10,4 & 8,5 & 6 & 12,4 \\
\hline \hline & 2,3 & 0,6 & 2,2 & 0,7 & 0,5 & 0 & 0,7 \\
\hline \hline total & 44 & 176 & 134 & 414 & 201 & 167 & 145 \\
\hline \hline
\end{tabular}

1: Inactifs; 2: Professions libérales, administrateurs, officiers militaires; 3 :Marchands, négociants ; 4 : Patrons et salariés de l'artisanat ; 5 : Laboureurs ; 6 :Salariés ; 7 : Pères inconnus.

Les prénoms très longs à quatre unités ou plus ont presque disparu, ce qui traduirait l'effacement du modèle aristocratique; la proportion de prénoms à trois unités est divisée par deux : voisine de $40 \%$ dans les élites, elle est ramenée à $20 \%$ environ ici. Même dans les catégories populaires où on trouvait $17,4 \%$, nous sommes maintenant au-dessous de $10 \%$. Inversement les prénoms courts repassent la barre des $25 \%$, et même $40 \%$ pour les milieux les plus modestes. Le mouvement est net et cette fois grande différence avec les "prénoms révolutionnaires" - il intéresse toutes les couches de la société. Quelle fraction de la population est concernée? Le graphique fournit peut-être une réponse approximative : la fréquence des prénoms courts passant de 25 à $45 \%$, c'est un cinquième environ des Rennais qui auraient modifié leur comportement. 
Cela est un minimum puisque n'entre pas dans cette évaluation la proportion de prénoms longs ou très longs ramenés à deux unités.

De manière cohérente, toutes les catégories sociales retrouvent des prénoms plus longs après 1795 .

Tableau $n^{\circ} 8$ : Longueur des prénoms (1796-1805)

\begin{tabular}{|c|c|c|c|c|c|c|c|}
\hline & 1 & 2 & 3 & 4 & 5 & 6 & 7 \\
\hline une unité & 14,7 & 12,9 & 16,3 & 17 & 24,4 & 21,6 & 26,1 \\
\hline deux unités & 44,1 & 49,1 & 55,9 & 60,7 & 57,3 & 61,6 & 60,2 \\
\hline trois unités & 35,3 & 32,7 & 25,2 & 20,8 & 17,6 & 15,6 & 12 \\
\hline $\begin{array}{l}\text { quatre unités et } \\
\text { plus }\end{array}$ & 5,9 & 5,3 & 2,6 & 1,5 & 0,7 & 1,3 & 1,7 \\
\hline total & 272 & 796 & 612 & 2338 & 988 & 713 & 1000 \\
\hline
\end{tabular}

1: Inactifs ; 2: Professions libérales, administrateurs, officiers militaires; 3 :Marchands, négociants ; 4 : Patrons et salariés de l'artisanat ; 5 : Laboureurs ; 6 : Salariés ; 7 : Pères inconnus.

Au vrai, il n'y a pas exactement retour à la situation antérieure. En particulier, les prénoms très longs ne réoccupent pas tout le terrain qu'ils avaient conquis. Sur ce plan, l'an II aura marqué une rupture, le modèle aristocratique a définitivement disparu, ce que des études portant sur le xixesiècle devront confirmer. Les prénoms à trois unités sont également moins nombreux qu'avant 1792. En revanche, ceux qui ont deux unités s'imposent comme jamais ils ne l'avaient fait auparavant. En tout état de cause, le retour aux prénoms courts n'aura été qu'un épisode, partagé par tout le corps social.

Il faudrait maintenant en dire le sens. Une première hypothèse serait que le raccourcissement des prénoms serait la conséquence mécanique d'amputations qui trouvent leur explication dans une analyse sémantique. Le prénom long reprenant volontiers les mêmes unités de base, que nous avons réunies sous le terme de fondamentales, leur recul aurait suffit à diminuer la longueur du prénom. Or le repli de ces fondamentales en 1794, examiné plus haut, était très modeste, et bien incapable d'expliquer la recrudescence importante des prénoms simples qui se produit au même moment. Socialement, le repli des fondamentales est limité à quelques groupes sociaux alors que toutes les catégories de Rennais participent au raccourcissement du prénom. Il est également difficile d'incriminer l'absence de parrains et de marraines lors de la déclaration à l'état civil : le phénomène aurait démarré plus tôt et se serait prolongé plus longtemps. D'autres explications sont donc indispensables.

La concomitance avec le développement des "prénoms révolutionnaires" ne permet plus d'écarter l'explication par la conjoncture politique. Le prénom long est-il "aristocratique"? Le plus simple serait de prendre en compte la différence de comportement selon les catégories sociales, en prenant le mot dans ses deux sens. $\mathrm{Au}$ premier, il désigne la noblesse celle-ci n'a sans doute plus le privilège de porter seule ce 
type de noms mais c'est encore elle qui porte les plus longs. Au deuxième sens, celui que les Sans-Culottes lui donnent, il vise tous ceux qui contreviennent à l'idéal d'une égalité de petits propriétaires; or les prénoms longs sont à Rennes la marque de l'opulence et des élites. Dès lors, il serait possible d'attribuer à ce prénom long, et donc à son raccourcissement, un sens politique.

Cherché deux fois, à Falaise et à Rennes ${ }^{26}$, ce signal original qu'est le raccourcissement du nom de naissance a été trouvé deux fois; dans chaque cas, il l'a été sur des lieux où les "prénoms révolutionnaires" sont peu nombreux, dans une chronologie qui les met en rapport étroit avec eux. Diminuer le nombre d'unités dans un prénom à Rennes au début de 1794 n'a certes pas le caractère d'une adhésion claire aux valeurs de la Révolution jacobine, comme les Brutus ou les Dix-Août le manifestent ostensiblement. Est-ce seulement une attitude de prudence, particulièrement bienvenue pour les élites qui prêtent le flanc aux accusations de la sans-culotterie et passent pour "aristocrates" ? Mais le geste est fait même par ceux que leur condition sociale protège de ce risque. Est-ce alors un compromis passé entre un idéal social neuf et le respect du registre chrétien et des liens familiaux traditionnels, qui gouvernaient depuis longtemps le choix du nom de l'enfant? Il y a de fortes chances, en réalité, pour que l'explication ne soit pas purement politique ou religieuse; elle peut être culturelle au sens le plus large. L'évolution du système prénominal au xviiiesiècle n'aurait pas préparé la masse des Rennais à se servir du prénom pour en faire l'affichage de valeurs politiques, alors que les élites avaient, elles, déjà assimilé cet usage, comme le président de la noblesse aux États de la province, ou le maire de la ville, en ont donné des exemples spectaculaires. Le refus d'un emploi massif des fondamentales et la place donnée aux références historiques auraient préparé la floraison des "prénoms révolutionnaires".

L'importance de ces derniers en un lieu devrait alors traduire la combinaison, dans des proportions qu'il faudrait établir à chaque fois, d'un degré d'engagement politique et d'un stade de l'évolution du système prénominal antérieure à la Révolution. Il est trop tôt pour conclure de manière définitive ou assurée. Au moins est-il indispensable de prendre en compte tous les signes que donne un système prénominal. Ils sont plus nombreux que ceux qui ont été évoqués ici : il faut encore songer aux études de la dispersion des prénoms, de leur hiérarchisation.

\section{ANNEXES}

Annexe : Grille de lecture des prénoms, à Rennes, entre 1785 et $1805^{27}$. Nombre total de références : 24010

Fondamentales

01 : Jean (3071) ; Joseph (1534); Marie (3331) ; Pierre (2992).

02 : François (2442); Julien (1628); Louis (1339).

03 : Baptiste, Jean-Baptiste (475). 
Références chrétiennes.

Anges, Évangiles.

11 : Anges : Ange (175); Archange (2); Gabriel (140); Michel (484)).

12 : Apôtres : André (153) ; Barthélémy (26) ; Jacques (627) ; Matthieu (40); Philippe(63) ;

Simon (53); Thomas (147).

13 : Apôtres (époque de Paul) : Barnabé (10); Étienne (165); Luc (26); Marc (70);

Paul(135); Siméon (11); Tite (1).

14 : Divers : Balthazar (2); Gaspard (17) ; Lazare (2); Melchior (1) ; Zacharie (3).

Saints.

21 : Saints légendaires, à pouvoirs miraculeux : Adrien (13); Antoine (182);

Christophe (26) ; Denis (23) : Eustache (18) ; Georges (108) ; Gilles (164) ; Guy (50); Martin

(55); Nicolas (275); Roch (6); Sébastien (36).

22 : Pères et docteurs de l'Église : Ambroise (80); Anselme (8); Athanase (3) ;

Augustin(165) ; Bernard (27) ; Bonaventure (12); Chrysostome (1) ; Grégoire (6) ; Hilaire (9) ;

Isidore (28) ; Jérôme (28).

23 : Antiquité : Agapit (1) ; Agathon (4); Alban (2) ; Amateur (1); Anatole (1); Antère(1);

Babylas (1) ; Blaise (3) ; Bon (2) ; Brice (2) ; Bénigne (6) ; Calixte (1) ; Chrysogone (1) ; Clair (3) ;

Clément (30) ; Cyprien (7) ; Cyr (1); Damase (3); Damien (1); Delphin (1); Donatien (1) ;

Eudoxe (1) ; Eusèbe (4) ; Fabien (2) ; Ferréol (1) ; Firmin (5) ; Flavien (1) ; Gatien (3) ; Germain

(15) ; Gervais (6) ; Gordien (2) ; Gratien (1) ; Hilarion ( 7) ; Honorat (3) ; Justin (1) ; Laurent

(183) ; Léonce (1) ; Léonide (1) ; Malchus (1) ; Marcel (1) ; Marcellin (10); Marin (10) ;

Mathurin (562) ; Maurille (5) ; Maxime (2) ; Maximilien (2) ; Pamphile (1) ; Patrice(7) ; Paulin

(3) ; Polycarpe (1); Ponce (1); Pélage (6); Romain (3); Saturnin (10) ; Second (1); Sylvestre

(3) ; Tiburce (1) ; Télesphore (1) ; Valentin (6); Valérien (1) ; Vital (1).

24 : Saints du Moyen Âge jusqu'à l'an Mil : Amand (81); Arnoult (1); Aubin (5);

Benoit(15) ; Boniface (2) ; Claude (100) ; Césaire (1) ; Elphège (1) ; Éleuthère (3) ; Éloi (3) ;

Exupère (1) ; Fiacre (2) ; Florent (13) ; Florentin (5) ; Fulgence (6) ; Gérard(1) ; Géraud (1)

Herménégild (2) ; Hubert (6) ; Just (1) ; Lambert (2) ; Landry (1) ; Liber (1) ; Lubin (1) ; Léger

(1) ; Léonard (11) ; Magloire (2) ; Médard(1) ; Médéric (2) ; Omer (1) ; Ouen (1) ; Philibert (5) ;

Rémi (7) ; Sulpice (2) ; Sylvain (7) ; Séverin (1); Sévère (4); Ulric (1).

25 : Saints depuis l'an Mil : Albert (7); Albéric (3); Anthelme (4); Avertin (1);Bernardin(4); Bertrand (44) Bruno (3); Cajétan (1) ; Célestin (25); Dominique(47); Edme (1); Edmond (8) ;

Ernest (4) ; Gautier (2) ; Gaëtan (3) ; Gilbert (15) ; Ignace (6) ; Népomucène (1) Pie (1) ;

Séraphin (1); Xavier, François-Xavier (10).

Références chrétiennes diverses.

31 : Noms bibliques : Abraham (4) ; Adam (1) ; Benjamin (19) ; Daniel (5) ; David (1) ; Élie

(9) ; Isaac (13) ; Jacob (2) ; Jérémie (1); Salomon (3).

32 : Divers : Anonyme (1) ; Bénit (4) ; Dieu (1) ; Dieudonné (2) ; Emmanuel (104) ; Esprit(14);

Noël (70) ; Pascal (29) ; Sauveur (2) ; Toussaint (29) ; Trinité (1).

Références historiques et morales.

41 : Mythologie, littérature ancienne : Achille (12); Adonis (1); Alcide (3); Ariste (1); Hercule (1) ; Hippolyte (101); Hyacinthe (80); Lucien (3) ; Mars (1) ; Narcisse (1) ; Tacite (1) ; Théagène (1); Térence (3). 
42 : Littérature médiévale, sciences : Arthur (3); Candide (1) ; Émile (23) ; Érasme (2); Euler (1) ; Olivier (185); Renaud (1) Roland (4); Tom (1).

Références historiques.

51 : Antiquité : Alexandre (154); Antonin (2); Aristide (16); Aristote (1); Auguste (168) ; Camille (2) ; Carolus (1) ; Constantin (1); César (10); Dymas (1); Fabius (1); Hannibal (1) ; Jules (7) ; Léo (1) ; Marius (2) ; Maxence (1) ; Octavius (1) ; Paul-Émile (4) ; Philogène (4) ; Scipion (1) ; Timoléon (2); Victor (106).

52 : Monde byzantin : Alexis (88); Basile (1); Léon (4); Maurice (33) ; Nicéphore (1); Théodore (70); Théophile (20).

53 : Moyen Âge : Alfred (18); Amaury (2) ; Arnaud (3); Charlemagne (1) ; Didier (3) ; Édouard (47) ; Geffroy (1) ; Godefroy (16) ; Raoul (13) ; Raymond (7) ; Richard (5) ; Robert (35) ; Sigismond (1); Thibaut (1).

54 : Époque contemporaine : Adolphe (68); Alphonse (6); Amédée (14); Casimir (8); Charles (504); Christian (1) ; Eugène (38) ; Ferdinand (7) ; Frédéric (84) ; Gustave(7); Henri (135) ; Ladislas (1); Rodolphe (12); Stanislas (15).

55 : "Prénoms révolutionnaires" : Bara (1); Brutus (5); Buis (1); Canon (1);Carrier(2); Citoyen Français (1) ; Coq (1) ; Dix Août (1) ; Égalité (3) ; Fargeau (1) ; Fleurus (3) ; Floréal (2) ; France (1) ; Franklin (1) ; Fédéré (3) ; Gracchus (1) ; Jasmin(1) ; La Montagne (1) ; Lespérance (1) ; Liberté (1) ; Marat (1) ; Patrie (1) ; Patriophile (3) ; Raison (2) ; Regulus (3) ; Romarin (2) ; Républicain (1) ; Révolution(1) ; Scaevola (3) ; Tell, Guillaume Tell (3) ; Thermidor (1) ; Troëne (1); Vent (1); Vertu (2); Viala (1); Vérité (1).

56 : Napoléon : Napoléon (12).

Prénoms à connotation morale.

61 : Noms de saints : Désiré (63) ; Fidèle (54); Félix (116); Honoré (15); Modeste (10);

Placide (7) ; Prosper (56) ; Urbain (18).

62 : Autres noms à connotation morale : Aimé (148); Brillant (1); Constant (46); Fortuné (1) ; Pacifique (1) ; Parfait (1) ; Prudence (1) ; Prudent (9) ; Sincère (1).

Références bretonnes.

71 : Province : Alain (38); Bretagne (1); Judicaël (1) ; Rennes (1); Vitré (1).

72 : Saints bretons : Armel (11) ; Corentin (3) ; Hervé (4) ; Léonor (3) ; Malo (2) ; Renan(2)

Samson (2); Vincent (110); Yves (223).

Prénoms féminins.

81 : Adélaïde (1); Agathe (3) ; Aimée (2) ; Angèle (3) ; Angélique (1) ; Anne (329); Barbe (5); Bathilde (1) ; Blanche (2); Catherine (6) ; Claire (1) ; Constance (4) ; Céleste (2) ; Élisabeth (12) ; Éleonor (5) ; Émilie (2) ; Esther (4) ; Eulalie (1) ; Fleurie (1) ; Flore (1) ; Florence (1) ; Françoise (1) ; Félicité (12) ; Gabrielle (2) ; Geneviève (2) ; Hélène (6) ; Josèphe (2) ; Judith (1) ; Julie (1) ; Julienne (1) ; Magdelaine (6) ; Marguerite (27) ; Marthe (1); Mélanie (1) ; Nicole (1) ; Olive (2) ; Reine (3) ; Rosalie (21) ; Scolastique (1) ; Sophie (2) ; Suzanne (10) ; Suzon (2) ; Thérèse (13); Ursule (7); Victoire (2).

Divers.

Cas particuliers : Guillaume (406); René (643).

Prénoms germaniques : Aldebert (1) ; Armand (35); Aubert (1) ; Macé (1). Hypocoristiques : Manuel (1); Victorien (1); Yvon (4).

Prénoms étrangers : Salvador (1). 
Non ou mal identifiés : Aly (1) ; Anchere (1) ; Aristolaton (1) ; Barbeluard (1) ; Chéréole(1) ; Filiane (1) ; Frazy (1) ; Judoc (1) ; Mellandon (1) ; Préjent (1) ; Saphir(1) ; Solime (1) ; Suzin (1). Illisibles : (6).

\section{NOTES}

1.J.-P.LETHUILLIER, Idéologie et mentalités : l'essor de l'individualisme en Basse-Normandie, (1650-1850), thèse, université de Paris-I, 1993. À l'université Rennes2, nous avons également mené des recherches sur les prénoms en Haute-Bretagne, et dirigé plusieurs mémoires de maîtrise sur ce thème, pour la même période 1650-1850 (Denis Joncquemat, 1996; Frédéric Barbu, Laurence Cuzin, Christophe Guéguen, Arnaud Lemée, Sophie Pelhate, Sandrine Piel, 1997; Valérie Bertrand, Laurence Urien, 1998). En 1999, Denis LECOURTILLER a réalisé un mémoire portant sur la ville de Rennes : Les prénoms à Rennes de 1660 à 1840. Nous nous sommes également servi du mémoire de Nadine BERTHAU, Cornélie, Jasmin et Espoir du Monde... Les prénoms "révolutionnaires" en Ille-etVilaine (1790-1800), sous la direction d'A.Croix, 1995.

2.Il nous paraît dangereux de confondre sous le même terme le "prénom" et les éléments qui le constituent. Jean Pierre est un "prénom", Jean et Pierre sont des "unités prénominales". Nous conserverons cependant, par exception, l'expression "prénoms révolutionnaires", devenue classique, pour désigner les unités caractéristiques de l'an II.

3.Les corpus masculin et féminin ne se structurent pas de la même manière et, autant qu'on puisse le mesurer, réagissent différemment au choc révolutionnaire : à Nantes et en Loire-Atlantique, les prénoms des filles sont moins directement politiques que ceux des garçons : A. CRoIX et P. SCILBo, Brutus, Désir de la Paix et Jonquille. Les prénoms de la Révolution en Loire-Inférieure, Nantes-Histoire, 1993, p.37.

4.N.BERTHAU, Cornélie, Jasmin et Espoir du Monde... Les prénoms "révolutionnaires" en Ille-etVilaine (1790-1800).

5.Pour Nantes : A. CRoIX et P. SCILBO, ouvr. cit. Pour un sondage à l'échelle du pays, voir aussi l'article de R.Bange dans ce numéro des AHRF. On ne prend en compte ici, pour exprimer la comparaison, que le noyau dur des prénoms révolutionnaires.

6.J.MEYER (dir.) : Histoire de Rennes, Privat, 1792, p.316.

7.Un exemple de critique méthodique dans A. CroIX et P. SCILBO, ouvr. cité, p.10.

8.R.BANGE : Les prénoms de l'an II et les autres... op. cit.

9.J.-P.LETHUilLieR, "Prénoms et Révolution : enquête sur le corpus falaisien", Annales de Normandie, 1989, n4, pp.413-436.

10.Outre les expériences acquises sur d'autres sites, nous utilisons ici le mémoire de D.LECOURTILLER, Les prénoms à Rennes de 1660 à 1840.

11.En Haute-Bretagne, D.Lecourtiller et S.Pelhate relèvent à Rennes et à MartignéFerchaud, au sud de Rennes, des fréquences en augmentation dans la première moitié du siècle, jusqu'à 5 ou $6 \%$, stables ensuite à Romillé, à l'ouest de Rennes, S.Piel constate au contraire une fréquence très faible au xviIIesiècle (de l'ordre de $1 \%$,) n'augmentant que pendant la première moitié du xixesiècle.

12.En Normandie, Louis connaît au milieu du siècle un renouveau qui l'apparente à celui de Charles et au développement de Frédéric, Eugène, Stanislas, Adolphe, etc., soit 
l'ensemble des monarques et des chefs de guerre de l'Europe du temps. J.-P. LethUILLIER, ouvr. cité, p. 473 et pp.479-480.

13.Par unité fondamentale, il faut entendre une unité prénominale dont la place dans le corpus est accrue par sa reprise très courante comme élément d'un prénom long. Sa fréquence est alors déterminée, comme toute unité, par son "sens", mais aussi par l'importance plus ou moins grande des prénoms longs. Jean est typiquement dans ce cas de figure.

14.Guillaume et René peuvent-ils être considérés comme des références historiques ? On pourrait en convenir en Normandie et en Anjou (voir H.NEVEUX : "Du prénom Guillaume et des Normands au xviesiècle", dans Recueil d'études en hommage à Lucien Musset, Cahiers des Annales de Normandie $n^{\circ} 23$ ) mais en Haute-Bretagne il faudrait admettre une influence extérieure sur la région, par le biais de migrants par exemple. Ce n'est pas impossible : pour René, S.Pelhate relève des fréquences de l'ordre de $10 \%$ à MartignéFerchaud, aux confins de l'Anjou, tout au long du xviIIesiècle (voir aussi François LEBRUN : "Les prénoms dans une paroisse rurale de l'Anjou entre 1751 et 1797. Notes sur l'exploitation d'un fichier de famille", dans Le prénom. Mode et histoire, Éditions de l'EHESS, 1984, pp. 241-243). Guillaume pourrait aussi être un prénom plus fréquent au diocèse de Saint-Brieuc, où la cathédrale conserve le tombeau de saint Guillaume Pinchon - ce qui cesserait d'en faire une simple référence historique. D'autres prénoms sont susceptibles d'être dans le corpus rennais des marques régionales ou locales portées par des migrants. Cette question est liée à celle d'une structure géographique particulière dans laquelle des prénoms découpent sur la carte une aire d'influence nettement marquée, un peu comme Léonard en Limousin (L.PEROUAS,B. BARRIÈRE,J. BoutieR,J.-C. PeYRonNet,J. TRICARD, Léonard, Marie, Jean et les autres. Les prénoms en Limousin depuis un millénaire, Éditions du CNRS, 1984), à l'inverse de la Normandie qui ignore ce type de répartition géographique.

15.Le contenu de chacune des catégories apparaît en annexe.

16. À l'inverse de ce qu'on observe à Falaise.J.-P. LETHUILLIER, "Prénoms et Révolution : enquête sur le corpus falaisien”, Annales de Normandie, 1989, pp. 413-436.

17.Mesurée en proportion des occurrences de Marie.

18. Tous les mémoires de maîtrise en témoignent, Marie devenant dès 1810, à Rennes, la première unité prénominale portée par les garçons. En ce sens, Marie est une fondamentale typique.

19.Pour Toussaint, octobre et novembre, puis le seul brumaire. Pour Noël, décembre puis nivôse.

20.Les observations des prénoms de la noblesse sont encore trop peu nombreuses. Les exemples normands montrent le rejet des fondamentales les plus banales : c'est au premier chef le cas de Jean.

21.Victor est une des références les plus complexes qui soient. Nom chrétien, son aspect "antique" offre un attrait à cette époque, comme Octavius, Carolus ou Philogène. Le rapport avec le nom féminin Victoire, et surtout avec le mot lui-même, et donc la guerre en cours, est également flagrant : Victor apparaît 23fois seulement de 1785 à 1792, mais 13 fois entre 1793 et 1795 et 70 fois entre 1796 et 1805.

22.La part des enfants trouvés est très faible : 28enfants trouvés et 1067enfants naturels.

23.A.TRABut, Égalité, Bourrache, Aristide et les autres. Les prénoms révolutionnaires dans le département des Côtes-du-Nord. 1789-1800, Vive 89 en Côtes-du-Nord, 1993, pp. 148-155. Voir aussiA. CROIX et P. ScILBO, ouvr. cité, pp.41-42. 
24.J.-P.LeThUillier, "Prénoms et Révolution : enquête sur le corpus falaisien”, Annales de Normandie, $\mathrm{n}^{\circ} 4,1989$, pp.413-436.

25. Ce point est délicat à établir en l'absence d'un dépouillement exhaustif des registres paroissiaux.

26.Il est bien possible que, dans l'Ouest, on puisse joindre Brest à ce lot. Les trois sondages effectués par Annie HENWOOD en 1789, 1794 et 1799 montrent que les prénoms de 1794 sont sensiblement plus courts : "Coriandre-Houlette ou Marie-Jeanne, MaratFinistère ou Jean-Marie. Étude des prénoms à Brest pendant la Révolution (1789-1799)”, Cahiers de l'Iroise, 1988, n³, pp. 145-160.

27.BÉNÉDICTINS DE RAMSGATE, Dix mille saints. Dictionnaire hagiographique, Brepols, 1991. Dictionnaire des saints bretons, Tchou, 1979.

\section{RÉSUMÉS}

La recherche des seuls "prénoms révolutionnaires" risque de n'éclairer que partiellement la réaction des systèmes prénominaux à l'événement politique, aux mutations de l'an II ; là où ils sont peu nombreux, le risque est même de laisser croire à la quasi-inertie des corpus. Cet article tente de montrer qu'il est souhaitable d'élargir la recherche, de s'intéresser à l'ensemble du stock des prénoms. À Rennes, où le registre chrétien des références prénominales résiste bien aux années de révolution, l'an II est le moment d'une flambée inaccoutumée de prénoms courts comme si, par opposition, le prénom long était "aristocratique".

\section{Male First Names in Rennes during the Revolution (1785-1805).}

Confining the search to « revolutionary first names » runs the risk of unevenly illuminating the way names react to political events, to the upheavals of YearII, and when they are few, it may give the impression that the corpuses are more or less inert. The author shows there is a need to widen the angle and include the entire stock of forenames. In Rennes, where the register of Christian names offers strong resistance to the years of Revolution, Year II yields an unusual crop of short forenames, as if, conversely, the long forename were « aristocratic ».

\section{INDEX}

Mots-clés : prénoms révolutionnaires, références chrétiennes, références historiques, prénoms courts

\section{AUTEUR}

JEAN-PIERRE LETHUILLIER

CRHISCO - Université de Rennes 2 